\title{
Comparison of Intelligent Code Acquisition for Sectorized Multi-Antenna CDMA in Downlink Mode
}

\author{
Ansgar Scherb, Volker Kühn and Karl-Dirk Kammeyer \\ Department of Communications Engineering \\ University of Bremen \\ Otto-Hahn-Allee \\ D-28359 Bremen, Germany \\ Email:\{scherb, kuehn, kammeyer\}@ant.uni-bremen.de
}

\begin{abstract}
This paper presents two different code acquisition schemes for sectorized multi-antenna CDMA systems in downlink mode. The so called "separated code optimization" (SCO) decomposes a two dimensional spreading code into its time and space component and treats each part separately. The SCO maximizes the signal to interference and noise ratio at each receiver. In contrast, the "joint code optimization" (JCO) does not distunguish between time and space component and acquires the code simultaneously. Both methods are evaluated by Monte Carlo simulations and their performance are compared.
\end{abstract}

\section{INTRODUCTION}

Observing recent publications in communications, two concepts are likely to become ingredients of future communication networks. On the one hand code division multiple access (CDMA) is a common technology in several 3G communication systems, on the other hand the use of multiple antennas is proposed to satisfy the demand on high data rates with low bandwidth.

Two classes of methods can be distinguished to exploit the possibilities given by multi-antenna arrays. Tarokh has introduced in 1998 space-time coding improving the quality of transmission by making use of spatial diversity effects [1]. Meanwhile, in this context a large number of contributions were published, e.g. [2] and [3]. Another class is the so called beamforming, focussing the transmit energy to the desired receiver. The principle of beamforming is to weight the transmit signals in such way that the receiver obtains a constructive superposition of different signal parts. Note that beamforming is not possible without some knowledge of the transmission channel at the transmitter. In [4] a comprehensive overview of beamforming techniques is given. Different proposals have been made that combine CDMA and multi-antenna techniques. A typical example is UMTS, where the Alamouti's space-time coding or closed loop beamforming are employed [5].

In the sequel, we direct our attention to the combination of beamforming and CDMA. The spreading code is usually designed enabling the receiver to extract the useful part out of the input signal in time domain. Moreover, beamforming can be interpreted as a kind of spatial signing. Therefore, the separation by spreading codes is assisted by the spatial component. In detail, for spatially separated users it may be suitable to distinguish them by their beamvectors, whereas for adjacent users the beamvectors should be nearly the same. Thus, they must be separated in time domain. The target of this paper is to examine how the load of a multi-user system can be increased by a combination of CDMA and SDMA techniques.

For further purpose we classify some properties:

- Consider the downlink in a static environment where fixed transmitter supports several mobile receivers.

- The transmitter has $N$ antennas, whereas the receivers just have one.

- The area which is covered by the base station can be divided into several sectors.

- The transmitter is able to locate each user as exact as necessary for assigning the user to a sector.

- The discrete time channel has zero mean and its covariance matrix, considering all possible channels between the transmitter and a receiver in a specific sector, should be known at the transmitter.

This paper is organized as follows: Section II introduces the system model. Section IV and V describes the SCO and JCO algorithm, respectively. Some Monte Carlo (MC) simulation results are presented in Section VI and finally a conclusion is presented in Section VII.

\section{System MOdEL}

The input of the $w$-th receiver is given by

$$
\mathbf{r}_{w}(m)=\mathbf{G}_{w} \mathbf{A} \mathbf{b}(m)+\mathbf{n}(m),
$$

where $\mathbf{r}_{w}(m)$ is a $(K \times 1)$-vector collecting all chips belonging to the $m$-th symbol, $K$ denotes the spreading factor, $\mathbf{b}(m)=\left[b_{1}(m), b_{2}(m), \cdots, b_{V}(m),\right]$ contains PSKsymbols of $V$ active users with mean $\left|b_{v}(m)\right|^{2}=1, \mathbf{A}=$ $\operatorname{diag}\left(\sqrt{a_{1}}, \sqrt{a_{2}}, \cdots, \sqrt{a_{V}}\right)$ is a diagonal matrix consisting of the root power of each user's signal and $\mathbf{n}(m)=$ $[n(1), n(2), \cdots, n(K)$,$] is i.i.d additive white gaussian noise$ 
(AWGN) with power $E\left\{|n(k)|^{2}\right\}=\sigma_{n}^{2}$. The signature matrix $\mathbf{G}_{w}$ is a $(K \times V)$-matrix defined by

$$
\begin{aligned}
\mathbf{G}_{w} & =\left[\mathbf{g}_{1, w}, \mathbf{g}_{2, w}, \cdots, \mathbf{g}_{V, w}\right] \\
& =\left[\mathbf{C}_{1} \mathbf{h}_{w}, \mathbf{C}_{2} \mathbf{h}_{w}, \cdots, \mathbf{C}_{V} \mathbf{h}_{w}\right]
\end{aligned}
$$

where $\mathbf{C}_{v}$ is a $(K \times N)$-matrix normalized to $\left\|\mathbf{C}_{v}\right\|_{F}^{2}=1$ containing the code of the $v$-th user's signal. Throughout this paper we consider a flat channel which can be expressed by the impulse response $\mathbf{h}_{v}=\left[h_{v, 1}, h_{v, 2}, \cdots, h_{v, N}\right]^{T}$ where $N$ denotes the number of antenna elements at the transmitter and $h_{v, n}$ is the fading gain of the channel from the $n$-th antenna of the base station to the $v$-th receiver. Note that the row and column space of $\mathbf{C}_{v}$ represents the space and time components of the code. Thus, time and space direction of the code can be treated independently as done in Section V. Usually, it can be assumed that $\mathbf{C}_{v}$ has rank 1 , although this is not madatory.

In order to treat space and time components of the code jointly, an alternative description of $\mathbf{g}_{v, w}$ can be given by

$$
\mathbf{g}_{v, w}=\tilde{\mathbf{H}}_{w} \tilde{\mathbf{c}}_{v}
$$

where $\tilde{\mathbf{c}}_{v}=\left[\mathbf{c}_{v}^{T}(1), \mathbf{c}_{v}^{T}(2), \cdots, \mathbf{c}_{v}^{T}(K)\right]^{T}$ is a $(N K \times 1)$-vector which is equivalent to $\mathbf{C}_{v}$ and

$$
\tilde{\mathbf{H}}_{w}=\left[\begin{array}{cccc}
\mathbf{h}_{w}^{T} & & & \mathbf{0} \\
& \mathbf{h}_{w}^{T} & & \\
& & \ddots & \\
\mathbf{0} & & & \mathbf{h}_{w}^{T}
\end{array}\right]
$$

is a $(K \times N K)$-matrix.

After matched filtering, the output of the $w$-th receiver can be obtained by

$$
y_{w}(m)=\overline{\mathbf{g}}_{w, w}^{H} \mathbf{r}_{w}(m)
$$

where

$$
\overline{\mathbf{g}}_{v, w}=\frac{\mathbf{g}_{v, w}}{\left\|\mathbf{g}_{v, w}\right\|^{2}}
$$

is the impulse response of the normalized matched filter. The recovered symbol $\hat{b}_{w}(m)$ may be obtained by quantizing suitably $y_{w}(m)$.

\section{A. Channel Characteristics}

The area covered by the base station should be split into $I$ different sectors, where $\mathbf{h}_{i}$ is the channel impulse response characterizing the transmission from base station to a receiver localized in the $i$-th sector. We assume $\mathbf{h}_{i}$ to be multivariate normal distributed with zero mean and covriance $\mathbf{R}_{i}=$ $\mathrm{E}\left\{\mathbf{h}_{i} \mathbf{h}_{i}^{H}\right\}$. Furthermore, all covariance matrices should be different, i.e. $\mathbf{R}_{i} \neq \mathbf{R}_{j}$ for $i, j \in\{1,2, \cdots, I\}$ and $i \neq j$. In order to exploit channel characteristics for the code design, we assume that the covariance matrix of each sector is known at the base station. Analogously, the covariance matrix in terms of space and time jointly as mentioned in (4) is given by $\tilde{\mathbf{R}}_{i}=E\left\{\tilde{\mathbf{H}}_{i} \tilde{\mathbf{H}}_{i}^{H}\right\}=\operatorname{diag}\left(\mathbf{R}_{i}^{H}, \cdots, \mathbf{R}_{i}^{H}\right)$.

\section{Problem Statement}

Generally, an upper bound for reliable data transmission for user $w$ is given by the well-known Shannon capacity. It can be expressed as function of the signal to noise ratio (SNR) of the $w$-th receiver

$$
C\left(\mathrm{SNR}_{w}\right)=\log _{2}\left(1+\mathrm{SNR}_{w}\right)
$$

In flat fading CDMA systems, the matched-filter output can only free of multiuser interference for $V \leq K$. Increasing the system load above $K$, additionally interference has to be taken into account. It may be easy to realize that in this case the system performance is influenced strongly by the code design. The capacity can be calculated by inserting the signal to interference and noise ratio (SINR) instead of SNR in (7), whereas a benchmark for the system performance is always given by the user which takes the worst SINR. The general topic of this paper is:

How has the code of all active users $\mathbf{C}_{w}$ or $\tilde{\mathbf{c}}_{w}$ in a multiantenna CDMA downlink scenario to be designed, such that the worst users SINR after matched filtering is maximized.

There are three degrees of freedom for designing suitable codes. As it will be shown in (8) each code word $\mathbf{C}_{w}$ can be decomposed into its time and space components weighted by the power term $\sqrt{a_{w}}$.

\section{Separated Code Optimisation (SCO)}

This approach treats the space and time components of the codes separately. Thus, the code can be decomposed into

$$
\mathbf{C}_{v}=\mathbf{c}_{v, \text { time }} \mathbf{c}_{i, \text { space }}^{H},
$$

where $\left|\mathbf{c}_{i \text {,space }}\right|^{2}=1$ and $\mid \mathbf{c}_{v}$,time $\left.\right|^{2}=1$ and user $v$ should be inside sector $i$. All users inside the same sector should have the same space code $\mathbf{c}_{i \text {,space, }}$, whereas the time code $\mathbf{c}_{v}$, time may differ for each user.

In order to share the resources among the users in a fair manner, a variable power allocation may be useful. Thus, the sum power of all users' signal should be fixed, but the specific power allocation may vary.

This section is subdivided into three parts. First, a heuristic approach for optimizing $\mathbf{c}_{v}$,time is explained. Part IV-B and IV-C deal with optimizing $\mathbf{c}_{i \text {,space }}$ and balancing the power, respectively. In order to get suitable code design, part IV-B and IV-C are repeated alternately until the algorithm converges to a global optimum as specified below.

1: Fix power allocation $\mathbf{A}$ and optimize $\mathbf{c}_{i \text {,space }}$.

2: Fix $\mathbf{c}_{i \text {,space }}$ and balance $\mathbf{A}$ such that channel capacity is splitted between all users in a fair manner and the contraint $\sum a_{i}=\rho$ is fulfilled, where $\rho$ is the available sum power.

Substantially, the results of these parts are based on [6]. This publication deals not with ST-CDMA but gives a solution for the SINR downlink beamforming problem. 


\section{A. Time Code}

Assume that the number of users inside a sector is limited by $K$. Furthermore, the user index $v$ is chosen such that all users inside a sector have consecutive indices. The task of $\mathbf{c}_{v}$,time is to ensure the separability of several signals being intended for users inside the same sector. For this purpose we use the same set of Walsh-Hadamard codes for each sector. In order to avoid giving two users in different sectors the same time code, the Walsh-Hadamard code is superimposed by a pseudo-noise (PN) scrambling code, which is unique for each sector. Assume that $K=2^{z}$ with $z \in \mathbb{N}$ and $\mathbf{t}_{l}=\left[t_{l}(1), \cdots, t_{l}(K)\right]^{T}$ is the $l$-th Walsh-Hadamard code word of length $K$ and $l=\bmod (v, K)$. Let $\mathbf{c}_{\mathrm{scramb}, i}=$ $\left[c_{\mathrm{scramb}, i}(1), \cdots, c_{\mathrm{scramb}, i}(K)\right]^{T}$ be the scrambling code of the $i$-th sector with $c_{\mathrm{scramb}, i}(k) \in\{+1,-1\}$, then the $v$-th user time code $\mathbf{c}_{v, \text { time }}=\left[c_{v, \text { time }}(1), \cdots, c_{y, \text { time }}(K)\right]^{T}$ can be obtained by

$$
c_{v, \text { time }}(k)=t_{l}(k) c_{\mathrm{scramb}, i}(k) .
$$

\section{B. Space Code}

First, we define the sum power $p_{i}$ by $p_{i}=\sum a_{v}$ where $v$ is an index of the set of all indices belonging to users localized in sector $i$ and $\mathbf{p}=\left[p_{1}, p_{2}, \cdots, p_{I}\right]^{T}$ and $\mathbf{C}_{\text {space }}=$ $\left[\mathbf{c}_{\text {space }, 1}, \mathbf{c}_{\text {space }, 2}, \cdots, \mathbf{c}_{\text {space }, I}\right]$.

Assume that the mean power of the remaining interference of the time code of signals being intended to different sectors is $E\left\{\left|\mathbf{c}_{\text {time }, v}^{H} \mathbf{c}_{\text {time }, w}\right|^{2}\right\}=1 / K$, where $v$ and $w$ are indices of users in different sectors. Therefore, the term $\operatorname{SINR}_{D L, j}$ which indicates the sum SINR of all users inside sector $j$ can be expressed as

$$
\operatorname{SINR}_{D L, j}\left(\mathbf{p}, \mathbf{C}_{\text {space }}\right)=\frac{p_{j} \mathbf{c}_{\text {space }, j}^{H} \mathbf{R}_{j} \mathbf{c}_{\text {space }, j}}{\sum_{i \neq j} \frac{p_{i}}{K} \mathbf{c}_{\text {space }, i}^{H} \mathbf{R}_{j} \mathbf{c}_{\text {space }, i}+\sigma_{n}^{2}}
$$

Calculating an optimization of the SINR term is not a trivial problem, because (10) depends on all available space codes and power levels. Fortunately, a duality between the SINR downlink and the SINR uplink beamforming problem was discussed in detail in [7], [8] and [6]. The expression for the uplink problem is

$$
\operatorname{SINR}_{U L, j}\left(\mathbf{p}, \mathbf{C}_{\text {space }}\right)=\frac{p_{j} \mathbf{c}_{\text {space }, j}^{H} \mathbf{R}_{j} \mathbf{c}_{\mathrm{space}, j}}{\sum_{i \neq j} \frac{p_{i}}{K} \mathbf{c}_{\mathrm{space}, j}^{H} \mathbf{R}_{i} \mathbf{c}_{\mathrm{space}, j}+\sigma_{n}^{2}}
$$

Eq. (11) just depends on $\mathbf{c}_{\mathrm{space}, j}$ and $\mathbf{p}$. A solution for $\mathbf{c}_{\mathrm{space}, j}$ can be found by calculating the eigenvector corresponding to the maximal eigenvalue of the pencil $\left(\mathbf{R}_{j}, \sum_{i \neq j} p_{i} \mathbf{R}_{i}+\sigma_{n}^{2} \mathbf{I}_{I}\right)$ [9]. Initializing $\mathbf{p}=[0, \cdots, 0]^{T}$ in the first iteration step, we will obtain beamvectors as space codes focussing as much energy as possible into the desired sector without regarding multiuser interference.

\section{Power Balancing}

The vector $\gamma \in \mathbb{R}_{+}^{I \times 1}$ which contains real positive values $\gamma_{i}$ for $i=1, \cdots, I$ is defined in such a way, that the relation between the target SINR of sector $i$ and sector $j$ can be expressed as

$$
\frac{\operatorname{SINR}_{D L, 1}}{\gamma_{1}}=, \cdots,=\frac{\operatorname{SINR}_{D L, I}}{\gamma_{I}}=\alpha,
$$

where $\alpha$ is a constant. As the SINR of each active user is nearly the same, we suggest to choose $\gamma$ such that $\gamma_{i}$ agrees with the number of active users in sector $i$. In order to get an expression for the power balancing, we define $\Psi\left(\mathbf{C}_{\text {space }}\right)$, containing the interference part of the signal, as function dependent on all space codes with $\Psi_{i, j}=\frac{1}{K} \mathbf{c}_{\mathrm{space}, j}^{H} \mathbf{R}_{i} \mathbf{c}_{\mathrm{space}, j}$ is its $i$-th row and $j$-th column element for $i \neq j$, and $\Psi_{i, j}=0$ for $i=j$. The matrix $\mathbf{D} \in \mathbb{R}_{+}^{I \times I}$, containing the useful signal part in the denominator of each diagonal element, is defined by $\mathbf{D}=\operatorname{diag}\left(\frac{\gamma_{1}}{\mathbf{c}_{\text {space }, 1}^{H} \mathbf{R}_{1} \mathbf{c}_{\text {space }, 1}}, \cdots, \frac{\gamma_{1}}{\mathbf{c}_{\text {space }, I}^{H} \mathbf{R}_{I} \mathbf{c}_{\text {space }, I}}\right)$. If the expression

$$
\frac{\mathbf{p}}{\alpha}=\mathbf{D} \mathbf{\Psi}\left(\mathbf{C}_{\text {space }}\right) \mathbf{p}+\sigma_{n}^{2} \mathbf{D} \mathbf{1} \text {. }
$$

is valid, the condition formulated in (12) is fulfilled [6].

In order to introduce the constraint of the sum power $\rho$, the 1.h.s. and r.h.s. of (13) are multiplied with $\mathbf{1}^{T}$

$$
\frac{1}{\alpha}=\frac{1}{\rho} \mathbf{1}^{T} \mathbf{D} \Psi\left(\mathbf{C}_{\text {space }}\right) \mathbf{p}+\frac{\sigma_{n}^{2}}{\rho} \mathbf{1}^{T} \mathbf{D} \mathbf{1} .
$$

Now with (13) and (14) the eigensystem

$$
\mathbf{\Upsilon} \overline{\mathbf{p}}=\frac{1}{\alpha} \overline{\mathbf{p}}
$$

can be obtained by defining

$$
\mathbf{\Upsilon}=\left[\begin{array}{cc}
\mathbf{D} \boldsymbol{\Psi}\left(\mathbf{C}_{\text {space }}\right) & \sigma_{n}^{2} \mathbf{D} \mathbf{1} \\
\frac{1}{\rho} \mathbf{1}^{T} \mathbf{D} \boldsymbol{\Psi}\left(\mathbf{C}_{\text {space }}\right) & \frac{\sigma_{n}^{2}}{\rho} \mathbf{1}^{T} \mathbf{D} \mathbf{1}
\end{array}\right]
$$

and $\overline{\mathbf{p}}=\left[\mathbf{p}^{T}, 1\right]^{T}$. The eigenvector corresponding to the

\begin{tabular}{|c|c|c|}
\hline 1 & : & Calculate the time code $\mathbf{c}_{w, \text { time }}$ as in Section IV-A \\
\hline 2 & : & $\begin{array}{l}\text { Initialize } \boldsymbol{\gamma} \text { with the number of active users in each sector, } \\
\overline{\mathbf{p}}=[\mathbf{0}, 1]^{H} \text { and the iteration counter } s=0\end{array}$ \\
\hline 3 & : & repeat \\
\hline 4 & : & $\begin{array}{l}\text { Fix } \mathbf{p}^{(s)} \text { and calculate a new set of beamforming } \\
\text { vectors } \mathbf{C}_{\text {space }}^{(s+1)} \text { by optimizing (11) }\end{array}$ \\
\hline 5 & : & Fix $\mathbf{C}_{\text {space }}^{(s+1)}$ and calculate $\mathbf{p}^{(s+1)}$ by solving (15) \\
\hline 6 & : & $s=s+1$ \\
\hline 7 & : & until (stopping criterion is fulfilled) \\
\hline 8 & : & $\begin{array}{l}\text { Determine the power } a_{w} \text { by dividing } p_{j} \text { by the number } \\
\text { of users inside sector } j\end{array}$ \\
\hline 9 & : & Obtain $\mathbf{C}_{p}$ from (8) \\
\hline
\end{tabular}
maximum eigenvalue is the solution of the power balancing problem.

\section{Complete Algorithm}

The algorithm is summarized in Table I. As a stopping criterion a threshold $\epsilon=\left\|\mathbf{C}_{\text {space }}^{(s)} \mathbf{p}^{(s)}-\mathbf{C}_{\text {space }}^{(s+1)} \mathbf{p}^{(s+1)}\right\|_{2}$ can be used, where $s$ is an iteration index. In most cases the SCO converges after 3-5 iterations.

TABLE I

SEPARATED CODE Optimization (SCO) 


\section{Joint Code Optimization (JCO)}

Up to now, we have dealt with time and space components of the code separately. In contrast to before, this approach does not distinguish between these constituents. Therefore, it may be suitable to use definition (3).

\section{A. Space and Time Code}

In order to avoid confusion let $w$ denote the index of the desired user which is localized inside the sector $j$ and $v$ denotes each other user which is localized in the sector $i$. Note that $i=j$ is not excluded. The interference power at the $w$-th receiver output localized in the $j$-th sector after matched filtering caused by the signal of $v$-th user is given by

$$
a_{v} \frac{\tilde{\mathbf{c}}_{v}^{H} \tilde{\mathbf{R}}_{j} \tilde{\mathbf{c}}_{w} \tilde{\mathbf{c}}_{w}^{H} \tilde{\mathbf{R}}_{j} \tilde{\mathbf{c}}_{v}}{\tilde{\mathbf{c}}_{w}^{H} \tilde{\mathbf{R}}_{j} \tilde{\mathbf{c}}_{w} \tilde{\mathbf{c}}_{w}^{H} \tilde{\mathbf{R}}_{j} \tilde{\mathbf{c}}_{w}}=a_{v} \frac{\tilde{\mathbf{c}}_{v}^{H} \mathbf{Q}_{w, w} \tilde{\mathbf{c}}_{v}}{\tilde{\mathbf{c}}_{w}^{H} \mathbf{Q}_{w, w} \tilde{\mathbf{c}}_{w}}
$$

As in Section IV we maximize the target function according to the uplink SINR:

$$
\widetilde{\operatorname{SINR}}_{U L, w}=\frac{a_{w} \tilde{\mathbf{c}}_{w}^{H} \tilde{\mathbf{R}}_{j} \tilde{\mathbf{c}}_{w}}{\sum_{v \neq w}^{V} \frac{a_{v}}{\tilde{\mathbf{c}}_{w}^{H} \tilde{\mathbf{R}}_{j} \tilde{\mathbf{c}}_{w}} \tilde{\mathbf{c}}_{w}^{H} \mathbf{Q}_{v, v} \tilde{\mathbf{c}}_{w}+\sigma_{n}^{2}}
$$

Note that (18) still depends on the set of all $\tilde{\mathbf{c}}_{v}$ and $a_{v}$ with $v=1, \cdots, V$. Therefore, a closed solution for this problem is still unknown. Without proof of convergence we suggest an iterative approach for optimizing (18). All variables denoted by the iteration index $s$ are based on $\tilde{\mathbf{c}}_{w}^{(s)}$. The updating rule is given in (19).

\section{B. Power Balancing}

As done in Section IV a fair allocation of power for all users can be achieved by power balancing. For that reason we replace the variables $\boldsymbol{\Upsilon}, \boldsymbol{\Psi}, \mathbf{D}, \mathbf{p}$ and $\alpha$ referred in Section IV. Instead, we use $\tilde{\mathbf{D}}=\operatorname{diag}\left(\frac{1}{\tilde{\mathbf{c}}_{1}^{H} \tilde{\mathbf{R}}_{1} \tilde{\mathbf{c}}_{1}}, \cdots, \frac{1}{\tilde{\mathbf{c}}_{V}^{H} \tilde{\mathbf{R}}_{I} \tilde{\mathbf{c}}_{V}}\right), \tilde{\mathbf{p}}=$ $\left[a_{1}, a_{2}, \cdots, a_{V}, 1\right]$ and $\tilde{\alpha}=\widetilde{\operatorname{SINR}}_{D L, 1}=, \cdots,=\widetilde{\operatorname{SINR}}_{D L, V}$. The matrix $\tilde{\boldsymbol{\Psi}}$ is defined such that

$$
\tilde{\Psi}_{v, w}=\frac{\tilde{\mathbf{c}}_{v}^{H} \mathbf{Q}_{w, v} \tilde{\mathbf{c}}_{v}}{\tilde{\mathbf{c}}_{v}^{H} \tilde{\mathbf{R}}_{v} \tilde{\mathbf{c}}_{v}}
$$

is the $v$-th column and $w$-th row element for $v \neq w$ and $\tilde{\Psi}_{v, w}=0$ for $v=w$. Furthermore,

$$
\tilde{\mathbf{\Upsilon}}=\left[\begin{array}{cc}
\tilde{\mathbf{D}} \tilde{\mathbf{\Psi}}(\tilde{\mathbf{U}}) & \sigma_{n}^{2} \tilde{\mathbf{D}} \mathbf{1} \\
\frac{1}{\rho} \mathbf{1} \tilde{\mathbf{D}} \tilde{\mathbf{\Psi}}(\tilde{\mathbf{U}}) & \frac{\sigma_{n}^{2}}{\rho} \mathbf{1}^{T} \tilde{\mathbf{D}} \mathbf{1}
\end{array}\right] .
$$

As derived in Section IV a solution is obtained by solving the special eigenvalue problem

$$
\tilde{\mathbf{\Upsilon}} \tilde{\mathbf{p}}=\frac{1}{\tilde{\alpha}} \tilde{\mathbf{p}} .
$$

where $\tilde{\mathbf{p}}$ is the eigenvector corresponding to the maximal eigenvalue.

Due to the high dimension of $\tilde{\Upsilon}$ the EVD will need a high computational effort. Furthermore, it may be desirably to perform this operation after each calculation of (19). Therefore, we suggest a simplified calculation of the eigenvectors based on the so called power method with

$$
\tilde{\mathbf{p}}^{(s+1)}=\frac{\tilde{\mathbf{\Upsilon}}^{(s)} \tilde{\mathbf{p}}^{(s)}}{\left\|\tilde{\Upsilon}^{(s)} \tilde{\mathbf{p}}^{(s)}\right\|^{2}},
$$

where $s$ is the iteration index [9].

\section{Complete Algorithm}

As stopping criterion a threshold similarly to Section IV can be employed. In most cases the CCO converges after 3-5 iterations. Another interesting point is the effort which must be spent for signal detection at the receiver. In order to apply the matched filter, either the receiver must have knowledge of the code and the CIR or of the convolutional product of both. Thus, either the receiver gets this information over a feedback or it may be conceivably to estimate the required filter coefficients by adapting it to a given pilot data sequence.

The proposed algorithm is summarized in Table II.

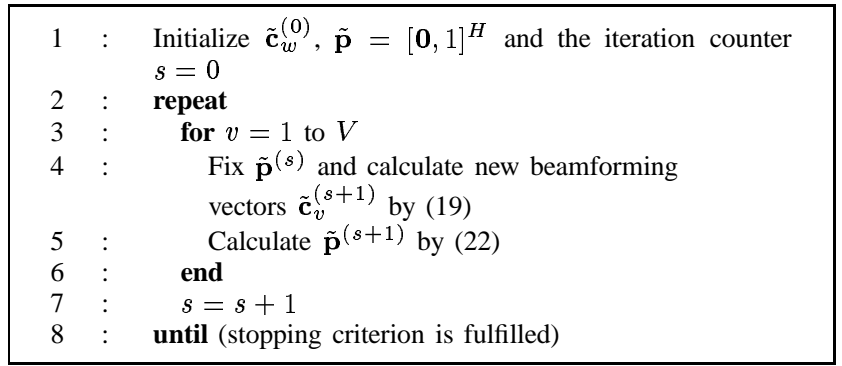

TABLE II

JOINT CODE OPTIMIZATION (JCO)

\section{MC SIMULATIONS}

In order to generate the covariance matrices we have considered a the base station with an uniform circular array (UCA) with 6 antennas and radius $\lambda / 4$. The area covered by the base station is divided into 6 uniformly distributed sectors with angle spread of $\pi / 3$. We obtain different covariance matrices $\mathbf{R}_{i}$, which have the same eigenvalues for $i=1, \cdots, 6$ denoted in Table III. In order to guarantee a passive channel $\operatorname{trace}\left(\mathbf{R}_{i}\right)=6$ holds. Note, that the maximal antenna gain, which can be achieved, corresponds to the maximal eigenvalue.

\begin{tabular}{|c|c|c|}
\hline & Eigenvalue & Eigenvalue $[\mathrm{dB}]$ \\
\hline \hline 1. & 5.3998 & 7.3238 \\
2. & 0.5832 & -2.3489 \\
3. & 0.0175 & -17.5690 \\
4. & 0.0004 & -33.4883 \\
5. & 0.0000 & -47.6195 \\
6. & 0.0000 & -54.6554 \\
\hline \multicolumn{3}{|c|}{ TABLE III }
\end{tabular}

Eigenvalues of $\mathbf{R}_{i}$

Fig. 1 shows the bit error rate (BER) vs. SNR for a system configured as specified in the caption. The graph denoted 


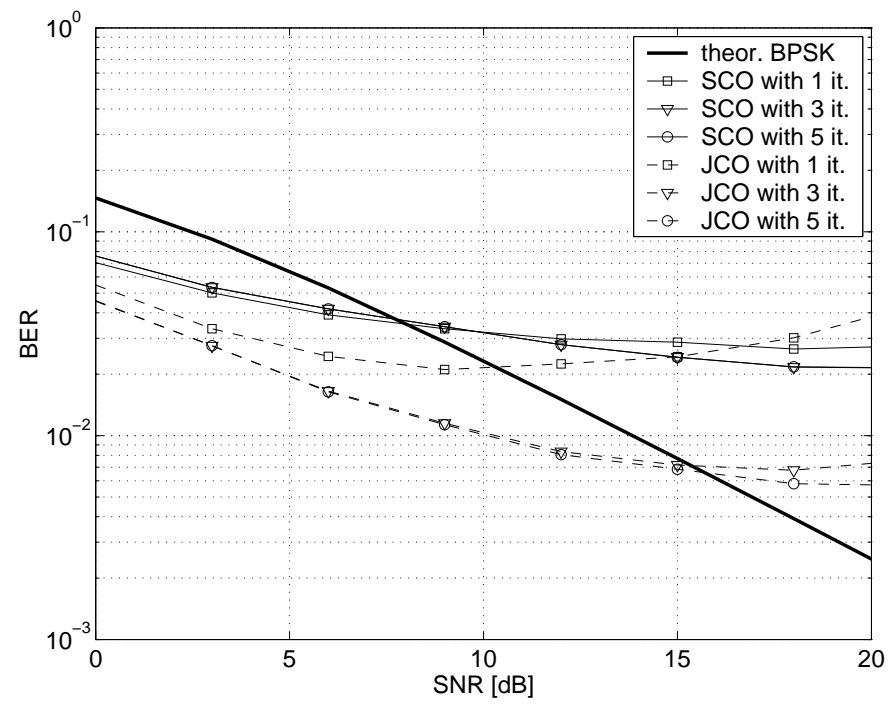

Fig. 1. BER vs. SNR for a system with spreading factor 8 and 24 active users in 6 sectors uniformly distributed

by "theor. BPSK" is the theoretical bit error rate, which is achieved for a single input single output (SISO) system over a 1-path Rayleigh fading channel. It can be seen that the performance of JCO for low SNR is approx. $7.3 \mathrm{~dB}$ better than the theoretical achievable BER of a Rayleigh fading BPSK transmission for all input SNR values. This represents exactly the antenna gain forecasted in Table III. However, SCO suffers much more from interference.

Evaluating the SNR vs. SINR graph in Fig. 2 the $7.3 \mathrm{~dB}$ gain of JCO can be found again. A noteworthy fact is that for low SNR on the one hand the later iterations of the SCO have a higher output SINR than the first, but on the other hand this does not affect the BER where the first iteration outperforms the latters. Remember that the first iteration of SCO represents straight forward beamforming without regarding MUI. An explanation may be given by the experimental observation that later iterations improve the output SINR for users transmitting over a strong channel but the output SINR of users with bad channels may suffer from it. Therefore, the main part of the BER is caused by the users with bad channels, whereas the improvement done by latter iterations do not preponderate in the face of BER.

\section{CONCLUSION}

We presented two different strategies for code acquisition for sectorized multi-antenna CDMA in downlink mode. SCO decomposes the code into its space and time components. Afterwards its time components are designed with WalshHadamard sequences inside a sector and scrambled by PN-

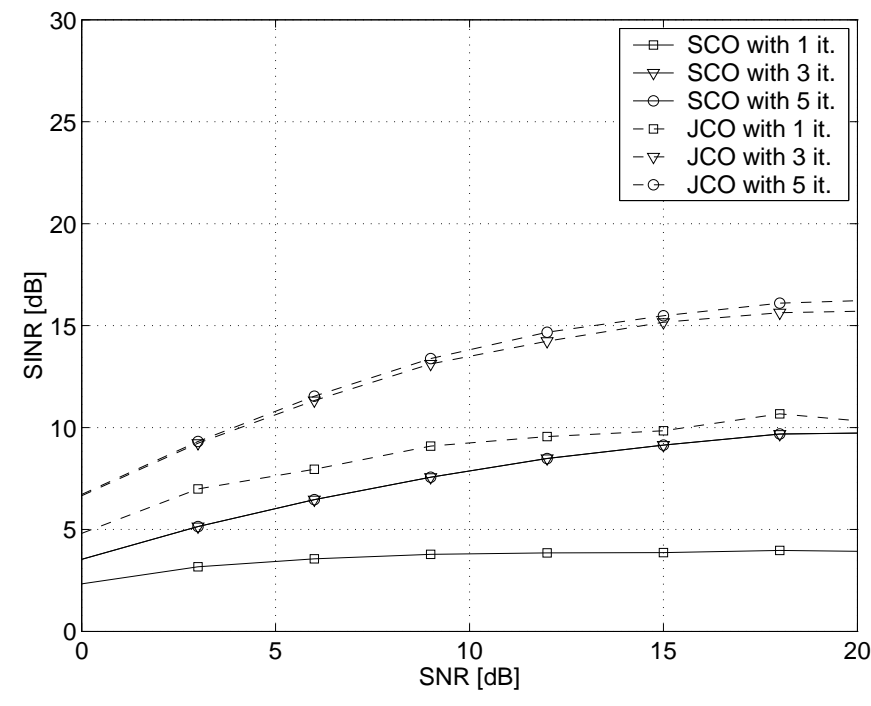

Fig. 2. SINR vs. SNR for a system with spreading factor 8 and 24 active users in 6 sectors uniformly distributed

sequences. The space component of the code is processed such, that the mean SNR at the receiver is maximum. In contrast to SCO, JCO jointly calculates the space and time components of the spreading code. A comparison of these algorithms by MC-simulations has demonstrated the superior performance of JCO. However, effects caused by estimation errors or transmission errors in the feedback channel in this simulation are not regarded.

\section{REFERENCES}

[1] N. Seshadri V. Tarokh and A.R. Calderbank. Space-Time Codes for High Data Rates Wireless Communications: Performance Criterion and Code Construction. IEEE Transactions on Information Theory, 44(2):744-765, March 1998.

[2] S.M. Alamouti. A Simple Transmit Diversity Technique for Wireless Communications. IEEE Journal on Select Areas in Communications, 16(8):1451-1458, October 1998.

[3] R. Gozali. Space-Time Coding and MIMO Channels - A Tutorial. www.mprg.ee.vt.edu/people/rgozali/tutorial.pdf, 2000

[4] D.H. Johnson and D.E. Dudgeon. Array Signal Processing: Concepts and Methods. Prentice Hall, Inc., 1992.

[5] European Telecommunications Standard Institute. Selection procedures for the choice of radio transmission technologies of the Universal Mobile Telecommunications System UMTS. DTR/SMG-50402, Version 0.9.4, December 1997.

[6] H. Boche and M. Schubert. Solution of the SINR Beamforming Problem. Conference on Information Science and Systems, pages 279-284, 2002.

[7] E. Visotsky and U. Madhow. Optimum Beamforming using Transmit Antenna Arrays. Proc. IEEE Vehicular Technology Conference, 1:851856, 1999.

[8] N. Jindal, S. Vishwanath, and A. Goldsmith. On the Duality between multiple access and broadcast channels. Proc Annual Allerton Conference, 2001.

[9] G.H. Golub and C.F. van Loan. Matrix Computation. John Hopkins University Press, 1989.

$$
\tilde{\mathbf{c}}_{w}^{(s+1)}=\arg \max _{\tilde{\mathbf{c}}_{w}} \frac{a_{w} \tilde{\mathbf{c}}_{w}^{H} \tilde{\mathbf{R}}_{j} \tilde{\mathbf{c}}_{w}}{\tilde{\mathbf{c}}_{w}^{H}\left(\frac{1}{\tilde{\left(\mathbf{c}_{w}^{(s)}\right)^{H} \tilde{\mathbf{R}}_{j} \tilde{\mathbf{c}}_{w}^{(s)}}}\left(\sum_{v=1}^{w-1} a_{v} \mathbf{Q}_{v, v}^{(s+1)}+\sum_{v=w+1}^{V} a_{v} \mathbf{Q}_{v, v}^{(s)}\right)+\sigma_{n}^{2} \mathbf{I}_{K N}\right) \tilde{\mathbf{c}}_{w}} .
$$

